\title{
The prime spectrum of the universal enveloping algebra of the 1-spatial ageing algebra and of $U\left(\mathfrak{g l}_{2}\right)^{*}$
}

\author{
V. Bavula and T. Lu
}

Communicated by A. P. Petravchuk

\begin{abstract}
A Bstract. For the algebras in the title, their prime, primitive and maximal spectra are explicitly described. For each prime ideal an explicit set of generators is given. An explicit description of all the containments between primes is obtained.
\end{abstract}

\section{Introduction}

Let $\mathbb{K}$ be a field of characteristic zero, $\mathbb{K}^{*}:=\mathbb{K} \backslash\{0\}, \mathbb{N}=\{0,1,2, \ldots\}$ and $\mathbb{N}_{+}=\{1,2, \ldots\}$.

The goal of the paper. Let $\mathcal{H}$ be the 3-dimensional Heisenberg Lie algebra and $\mathfrak{b}$ be the Borel subalgebra of $\mathfrak{s l}_{2}$. The semidirect products of Lie algebras $\mathfrak{s}=\mathfrak{s l}_{2} \ltimes \mathcal{H}$ and $\mathfrak{a}:=\mathfrak{b} \ltimes \mathcal{H}$ are called the Schrödinger algebra and the 1-spatial ageing algebra, respectively. Clearly, $\mathfrak{a} \subseteq \mathfrak{s}$. Let $U(\mathfrak{s})$ and $\mathcal{A}=U(\mathfrak{a})$ be the universal enveloping algebras of the Lie algebras $\mathfrak{s}$ and $\mathfrak{a}$. Then $\mathcal{A} \subseteq U(\mathfrak{s})$.

Let $\mathfrak{g l}_{2}$ be the Lie algebra of $2 \times 2$ matrices over $\mathbb{K}$ and $U\left(\mathfrak{g l}_{2}\right)$ be its universal enveloping algebra. The aim of the paper is for the algebras $\mathcal{A}$ and $U\left(\mathfrak{g l}_{2}\right)$ to classify their prime, primitive and maximal ideals

${ }^{*}$ Both authors were supported by the NSFC 11701190 . V. Bavula was supported by the Royal Society.

2020 MSC: 17B10, 16D25, 16D60, 16D70, 16P50.

Key words and phrases: prime ideal, maximal ideal, a universal enveloping algebra, prime spectrum. 
(Theorem 1, Corollary 1, Corollary 3, Theorem 2, Corollary 5 and Corollary 4.(1)). For each prime ideal an explicit (finite) set of generators is given. All the containments between primes are explicitly described, i.e., the Zariski-Jacobson topology on the spectra are described. In [6], the prime, completely prime, maximal and primitive ideals of the algebra $U(\mathfrak{s})$ are classified. Generating sets are given for all prime ideals of $U(\mathfrak{s})$ apart from an explicit set $\left\{\mathbb{I}_{n}^{\prime} \mid n \in \mathbb{N}_{+}\right\}$(see [6, Theorem 3.3]). Primitive ideals of $U(\mathfrak{s})$ with nonzero-central charge were described by Dubsky, Lü, Mazorchuk and Zhao [13] in the following way: Each such ideal is the annihilator of a simple highest weight $U(\mathfrak{s})$-module with nonzero central charge. The prime ideals of the quantum spatial ageing algebra are classified in [3]. Modules over the Schrödinger algebra are studied in [9, 12-14, 17, 19-21]. In [4], simple weight $\mathfrak{s}$-modules are classified.

The prime spectrum of the algebra $\mathcal{A}$. Recall that $\mathfrak{s l}_{2}=\mathbb{K} F \oplus$ $\mathbb{K} H \oplus \mathbb{K} E$ where $[H, E]=2 E,[H, F]=-2 F$ and $[E, F]=H$ and $\mathfrak{b}:=\mathbb{K} H \oplus \mathbb{K} E$ is its Borel subalgebra. The 3-dimensional Heisenberg Lie algebra $\mathcal{H}=\mathbb{K} X \oplus \mathbb{K} Y \oplus \mathbb{K} Z$ where $[X, Y]=Z$ and $Z$ is a central element of $\mathcal{H}$. As an abstract algebra, the algebra $\mathcal{A}$ is generated by the elements $H, E, X, Y$ and $Z$ subject to the defining relations:

$$
\begin{aligned}
& {[H, E]=2 E, \quad[H, X]=X, \quad[H, Y]=-Y,} \\
& {[E, X]=0, \quad[E, Y]=X, \quad[X, Y]=Z \text {, }}
\end{aligned}
$$

and $Z$ is a central element of $\mathcal{A}$. Recall that the algebra $\mathcal{A}$ is the subalgebra of $U(\mathfrak{s})$ generated by the elements $H, E, X, Y$ and $Z$ and the algebra $\mathcal{A}$ is isomorphic to the enveloping algebra $U(\mathfrak{a})$ of the solvable Lie subalgebra $\mathfrak{a}$. By [18, Theorem 8.3.36], all prime ideals of the algebra $\mathcal{A}$ are completely prime.

Let $H^{\prime}:=H+Z^{-1} X Y-\frac{1}{2}$ and $E^{\prime}:=E-\frac{1}{2} Z^{-1} X^{2}$. Then $\left[H^{\prime}, E^{\prime}\right]=$ $2 E^{\prime}$. It was proved in [6] that the algebra $\mathcal{A}_{Z}$ (the localization of $\mathcal{A}$ at the powers of the central element $Z$ ) is a tensor product of three algebras

$$
\mathcal{A}_{Z}=\mathbb{K}\left[Z^{ \pm 1}\right] \otimes \mathbb{K}\left[H^{\prime}\right]\left[E^{\prime} ; \sigma\right] \otimes A_{1}
$$

where $\mathbb{K}\left[H^{\prime}\right]\left[E^{\prime} ; \sigma\right]$ is a skew polynomial algebra where $\sigma\left(H^{\prime}\right)=H^{\prime}-2$ and $A_{1}:=\mathbb{K}\langle\mathscr{X}, Y\rangle$ is the Weyl algebra, $[\mathscr{X}, Y]=1$, where $\mathscr{X}:=Z^{-1} X$. The algebras $\mathcal{A}$ and $\mathcal{A}_{Z}$ are Noetherian algebras of Gelfand-Kirillov dimension 5 .

Let $\mathcal{E}:=E^{\prime} Z=E Z-\frac{1}{2} X^{2}$ and $\mathcal{H}:=H^{\prime} Z+\frac{1}{2} Z=H Z+X Y$. Then $\mathcal{E}$ is a normal element of the algebra $\mathcal{A}$, i.e., $\mathcal{E} \mathcal{A}=\mathcal{A} \mathcal{E}$. In fact, $\mathcal{E} H=(H-2) \mathcal{E}, \mathcal{E} E=E \mathcal{E}, \mathcal{E} Y=Y \mathcal{E}, \mathcal{E} X=X \mathcal{E}$ and $\mathcal{E} Z=Z \mathcal{E}$. 
Let $\mathcal{P}:=\{\mathfrak{p} \in \operatorname{Spec}(\mathbb{K}[Z, \mathcal{H}]) \mid$ ht $(\mathfrak{p})=1\}$. Recall that every height 1 prime ideal $\mathfrak{p}$ is generated by a simple irreducible polynomial which is unique up to multiplicative non-zero constant. Let $\overline{\mathcal{A}}:=\mathcal{A} /(\mathcal{E})$ and $\widetilde{\mathcal{A}}:=\mathcal{A} /(\mathcal{E}, Z)$. For an element $a \in \mathcal{A}$, let $\bar{a}=a+(\mathcal{E}) \in \overline{\mathcal{A}}$ and $\tilde{a}=$ $a+(\mathcal{E}, Z) \in \widetilde{\mathcal{A}}$.

In the diagram below, $\prod_{\mathfrak{p}}^{\mathfrak{q}}$ means $\mathfrak{p} \subsetneq \mathfrak{q}$, and $\underset{\dot{G}}{\stackrel{H}{\vdots}}$ means obvious inclusions between the sets of primes in sets $G$ and $H$.

Theorem 1. The set of prime ideals of the algebra $\mathcal{A}$ (together with all possible containments between the primes) is given in the following diagram:

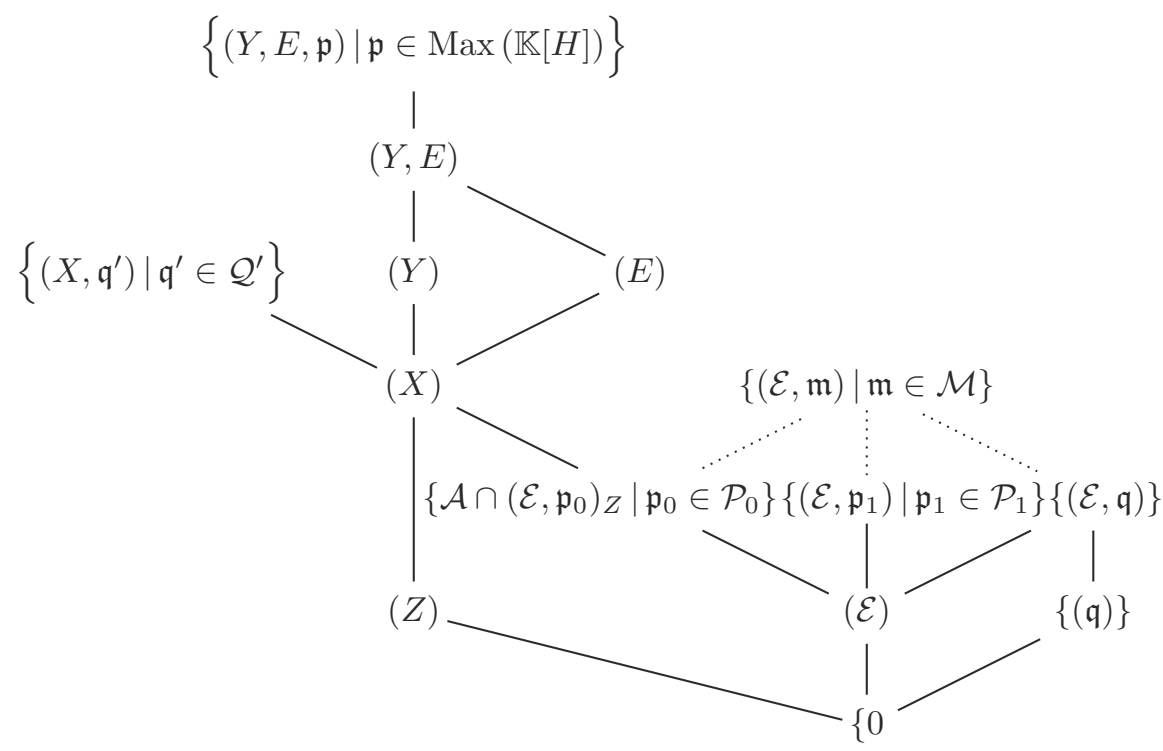

(2)

where $\mathfrak{q}^{\prime} \in \mathcal{Q}^{\prime}:=\operatorname{Max}\left(\mathbb{K}\left[Z^{\prime}\right]\right) \backslash\left\{\left(Z^{\prime}\right)\right\}$ and $Z^{\prime}:=E Y^{2}, \mathfrak{q} \in \mathcal{Q}:=$ $\operatorname{Max}(\mathbb{K}[Z]) \backslash\{(Z)\}, \mathfrak{p}_{0} \in \mathcal{P}_{0}:=\left\{\mathfrak{p}_{0}^{\prime} \in \mathcal{P} \mid \mathfrak{p}_{0}^{\prime} \neq(Z), \mathfrak{p}_{0}^{\prime} \subseteq(Z, \mathcal{H})\right\}$, $\mathfrak{p}_{1} \in \mathcal{P}_{1}:=\mathcal{P} \backslash\left(\mathcal{P}_{0} \sqcup\left\{\left(\mathfrak{q}^{\prime}\right) \mid \mathfrak{q}^{\prime} \in \operatorname{Max}(\mathbb{K}[Z])\right\}\right)$ and the set $\mathcal{M}:=\{\mathfrak{m} \in$ $\operatorname{Max}(\mathbb{K}[Z, \mathcal{H}]) \mid Z \notin \mathfrak{m}\}$. Furthermore, let $\mathfrak{p}_{0} \in \mathcal{P}_{0}$, then $\mathfrak{p}_{0}=(f)$ for some irreducible polynomial $f=a_{0}(Z)+a_{1}(Z) \mathcal{H}+\cdots+a_{n}(Z) \mathcal{H}^{n} \in$ $\mathbb{K}[Z, \mathcal{H}] \backslash(\mathbb{K} \cup \mathbb{K} Z)$ with $a_{0}(0)=0$ and (see Theorem 3.(2)), then

$$
\mathcal{A} \cap(\mathcal{E}, f)_{Z}= \begin{cases}\left(\mathcal{E}, \bar{f}_{\mathrm{red}}\right), & \text { if } \tilde{f}_{\mathrm{red}} \notin(X), \\ \left(\mathcal{E}, \bar{f}_{\mathrm{red}}, Z^{-1} \bar{f}_{\mathrm{red}} X\right), & \text { if } \tilde{f}_{\mathrm{red}} \in(X),\end{cases}
$$

see (9) for the definition of $f_{\text {red. }}$. 
The prime spectrum of $\boldsymbol{U}\left(\mathfrak{g l}_{2}\right)$. The Lie algebra $\mathfrak{g l}_{2}$ is the direct product of the Lie algebra $\mathfrak{s l}_{2}$ and a 1 -dimensional Lie algebra $\mathbb{K} Z$. So, $Z$ is a central element of the algebra $\mathcal{U}:=U\left(\mathfrak{g l}_{2}\right)$. Clearly, $\mathcal{U}=U\left(\mathfrak{s l}_{2}\right) \otimes \mathbb{K}[Z]$ is a tensor product of algebras. Hence, $Z(\mathcal{U})=Z\left(U\left(\mathfrak{s l}_{2}\right)\right) \otimes \mathbb{K}[Z]=\mathbb{K}[\Delta, Z]$ where $\Delta=4 F E+H^{2}+2 H$ is the Casimir element of $U\left(\mathfrak{s l}_{2}\right)$.

Theorem 2. Let $\mathbb{K}$ be a field of characteristic zero. Then the set of prime ideals of the algebra $\mathcal{U}=U\left(\mathfrak{g l}_{2}\right)$ and all the containments between primes are given in the following diagram:

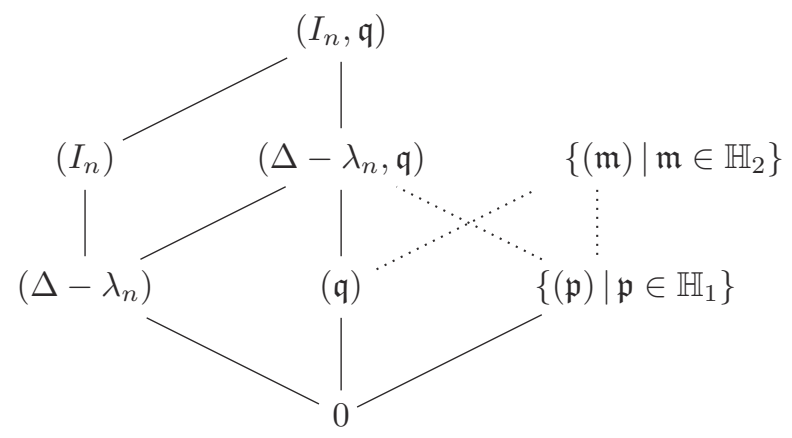

where $n \in \mathbb{N}_{+}, \lambda_{n}:=n^{2}-1, I_{n}$ is the annihilator of the simple $n$ dimensional $U\left(\mathfrak{s l}_{2}\right)$-module, $\mathfrak{q} \in \operatorname{Max}(\mathbb{K}[Z])$, and the set $\mathbb{H}_{1}:=\{\mathfrak{p} \in$ $\operatorname{Spec}(\mathbb{K}[Z, \Delta]) \mid \operatorname{ht}(\mathfrak{p})=1, \mathfrak{p} \cap \mathbb{K}[Z]=0, \mathfrak{p} \neq\left(\Delta-\lambda_{n}\right)$ for all $\left.n \in \mathbb{N}_{+}\right\}$, and $\mathbb{H}_{2}:=\operatorname{Max}(\mathbb{K}[Z, \Delta]) \backslash\left\{\left(\Delta-\lambda_{n}, \mathfrak{q}\right) \mid n \in \mathbb{N}_{+}, \mathfrak{q} \in \operatorname{Max}(\mathbb{K}[Z])\right\}$.

\section{Prime ideals of the algebra $\mathcal{A}$}

In this section, classifications of prime, primitive and maximal ideals of the algebra $\mathcal{A}$ are given (Theorem 1, Corollary 3 and Corollary 1). Moreover, for each of the prime ideals of $\mathcal{A}$ an explicit set of generators is given.

For an algebra $R$, let $\operatorname{Spec}(R)$ be the set of its prime ideals. The set $(\operatorname{Spec}(R), \subseteq)$ is a partially ordered set (poset) with respect to inclusion of prime ideals. Each element $r \in R$ determines two maps from $R$ to $R$, $r \cdot: x \mapsto r x$ and $\cdot r: x \mapsto x r$ where $x \in R$. An element $a \in R$ is called a normal element if $R a=a R$.

Proposition 1 ([3]). Let $R$ be a Noetherian ring and $s$ be an element of $R$ such that $S_{s}:=\left\{s^{i} \mid i \in \mathbb{N}\right\}$ is a left denominator set of the ring $R$ and $\left(s^{i}\right)=(s)^{i}$ for all $i \geqslant 1$ (e.g., $s$ is a normal element such that $\left.\operatorname{ker}\left(\cdot s_{R}\right) \subseteq \operatorname{ker}\left(s_{R} \cdot\right)\right)$. Then $\operatorname{Spec}(R)=\operatorname{Spec}(R, s) \sqcup \operatorname{Spec}_{s}(R)$ where 
$\operatorname{Spec}(R, s):=\{\mathfrak{p} \in \operatorname{Spec}(R) \mid s \in \mathfrak{p}\}, \operatorname{Spec}_{s}(R):=\{\mathfrak{q} \in \operatorname{Spec}(R) \mid s \notin \mathfrak{q}\}$ and

(a) the map $\operatorname{Spec}(R, s) \rightarrow \operatorname{Spec}(R /(s)), \mathfrak{p} \mapsto \mathfrak{p} /(s)$, is a bijection with the inverse $\mathfrak{q} \mapsto \pi^{-1}(\mathfrak{q})$ where $\pi: R \rightarrow R /(s), r \mapsto r+(s)$,

(b) the map $\operatorname{Spec}_{s}(R) \rightarrow \operatorname{Spec}\left(R_{s}\right), \mathfrak{p} \mapsto S_{s}^{-1} \mathfrak{p}$, is a bijection with the inverse $\mathfrak{q} \mapsto \sigma^{-1}(\mathfrak{q})$ where $\sigma: R \rightarrow R_{s}:=S_{s}^{-1} R, r \mapsto \frac{r}{1}$.

(c) For all $\mathfrak{p} \in \operatorname{Spec}(R, s)$ and $\mathfrak{q} \in \operatorname{Spec}_{s}(R), \mathfrak{p} \nsubseteq \mathfrak{q}$.

Since $Z$ is a central element of $\mathcal{A}$, by Proposition 1 , we have the disjoint union

$$
\operatorname{Spec}(\mathcal{A})=\operatorname{Spec}(\mathcal{A} /(Z)) \sqcup \operatorname{Spec}\left(\mathcal{A}_{Z}\right)
$$

We identify the sets of prime ideals in (5) via the bijections given in the statements $(a)$ and $(b)$ of Proposition 1 . The factor algebra $\mathcal{A} /(Z)$ is studied in [7] where a classification of prime ideals of $\mathcal{A} /(Z)$ is given, see [7, Theorem 2.5]. By [7, Theorem 2.5], the set of prime ideals of $\mathcal{A}$ containing the element $Z$ contains precisely the ideals in (2) over $(Z)$. So, it remains to describe the set $\operatorname{Spec}\left(\mathcal{A}_{Z}\right)$, i.e., the set of prime ideals of $\mathcal{A}$ that do not contain the central element $Z$.

For an element $a \in \mathcal{A}_{Z}$, we denote by $(a)_{Z}$ the ideal of $\mathcal{A}_{Z}$ generated by the element $a$. Clearly, $(\mathcal{E})_{Z}=\left(E^{\prime}\right)_{Z}$. Let $\mathcal{A}_{Z, \mathcal{E}}$ be the localization of the algebra $\mathcal{A}_{Z}$ at the powers of the element $\mathcal{E}$. Notice that $E^{\prime}$ and $\mathcal{E}$ are normal elements of $\mathcal{A}_{Z}$. Clearly, $\mathcal{A}_{Z, E^{\prime}}=\mathcal{A}_{Z, \mathcal{E}}$. By Proposition 1 ,

$$
\operatorname{Spec}\left(\mathcal{A}_{Z}\right)=\operatorname{Spec}\left(\mathcal{A}_{Z} /(\mathcal{E})_{Z}\right) \sqcup \operatorname{Spec}\left(\mathcal{A}_{Z, \mathcal{E}}\right)
$$

Now, by (1), the factor algebra

$$
\mathcal{A}_{Z} /(\mathcal{E})_{Z} \simeq \mathbb{K}\left[Z^{ \pm 1}, H^{\prime}\right] \otimes A_{1}=\mathbb{K}\left[Z^{ \pm 1}, \mathcal{H}\right] \otimes A_{1}
$$

is a tensor product of algebras. By (1), the localized algebra

$$
\begin{aligned}
\mathcal{A}_{Z, \mathcal{E}} & =\mathbb{K}\left[Z^{ \pm 1}\right] \otimes \mathbb{K}\left[H^{\prime}\right]\left[E^{\prime \pm 1} ; \sigma\right] \otimes A_{1} \\
& =\mathbb{K}\left[Z^{ \pm 1}\right] \otimes \mathbb{K}\left[H^{\prime}\right]\left[\mathcal{E}^{ \pm 1} ; \sigma\right] \otimes A_{1}
\end{aligned}
$$

is a tensor product of algebras where the algebras $\mathbb{K}\left[H^{\prime}\right]\left[\mathcal{E}^{ \pm 1} ; \sigma\right]$ and $A_{1}$ are central simple algebras.

The algebra $\mathcal{A}$ is a Noetherian domain. $\operatorname{Let} \operatorname{Frac}(\mathcal{A})$ be its skew field of fractions. The next lemma describes the centres of the algebras $\mathcal{A}$ and $\operatorname{Frac}(\mathcal{A})$. 
Lemma 1. $Z(\mathcal{A})=\mathbb{K}[Z]$ and $Z(\operatorname{Frac}(\mathcal{A}))=\mathbb{K}(Z)$.

Proof. By (8), $Z\left(\mathcal{A}_{Z, \mathcal{E}}\right)=\mathbb{K}\left[Z^{ \pm 1}\right]$. Since $\mathbb{K}[Z] \subseteq Z(\mathcal{A}) \subseteq Z\left(\mathcal{A}_{Z, \mathcal{E}}\right) \cap \mathcal{A}=$ $\mathbb{K}\left[Z^{ \pm 1}\right] \cap \mathcal{A}=\mathbb{K}[Z]$, we have $Z(\mathcal{A})=\mathbb{K}[Z]$. The second equality in the lemma follows directly from (8).

Next, we represent the factor algebra $\mathcal{A} /(\mathcal{E})$ as an iterated Ore extension. It follows from this fact that $(\mathcal{E})_{Z} \cap \mathcal{A}=(\mathcal{E})$.

Lemma 2. $\mathcal{A} /(\mathcal{E}) \simeq \Lambda\left[Y ; \delta_{1}\right]\left[H ; \delta_{2}\right]$ is an iterated Ore extension over the commutative domain $\Lambda:=\mathbb{K}[E, X, Z] /(\mathcal{E})$ where the derivations $\delta_{1}$ and $\delta_{2}$ are defined in the proof. In particular, $(\mathcal{E})$ is a completely prime ideal of $\mathcal{A}$ and $(\mathcal{E})_{Z} \cap \mathcal{A}=(\mathcal{E})$.

Proof. Notice that $\mathcal{A}=\bigoplus_{i, j \in \mathbb{N}} \mathbb{K}[E, X, Z] Y^{i} H^{j}$. Since $\mathcal{E}$ is a normal element of $\mathcal{A}$, we have $(\mathcal{E})=\mathcal{E} \mathcal{A}=\bigoplus_{i, j \in \mathbb{N}} \mathcal{E} \mathbb{K}[E, X, Z] Y^{i} H^{j}$. Hence, $\mathcal{A} /(\mathcal{E}) \simeq \Lambda\left[Y ; \delta_{1}\right]\left[H ; \delta_{2}\right]$ where $\delta_{1}$ is the derivation of $\Lambda$ defined by the rule

$$
\delta_{1}(E)=-X, \delta_{1}(X)=-Z, \delta_{1}(Z)=0
$$

and $\delta_{2}$ is the derivation of the algebra $\Lambda\left[Y ; \delta_{1}\right]$ defined by the rule $\delta_{2}(E)=$ $2 E, \delta_{2}(X)=X, \delta_{2}(Z)=0$ and $\delta_{2}(Y)=-Y$.

Since $\mathcal{E}=E Z-\frac{1}{2} X^{2}$ is an irreducible polynomial in $\mathbb{K}[E, X, Z]$, the factor algebra $\Lambda=\mathbb{K}[E, X, Z] /(\mathcal{E})$ is a domain. Then the iterated Ore extension $\mathcal{A} /(\mathcal{E})$ is a domain, i.e., $(\mathcal{E})$ is a completely prime ideal of $\mathcal{A}$. Now, let $u \in(\mathcal{E})_{Z} \cap \mathcal{A}$. Then $Z^{i} u \in(\mathcal{E})$ for some $i \in \mathbb{N}$. Since $(\mathcal{E})$ is a completely prime ideal of $\mathcal{A}$ and $Z \notin(\mathcal{E})$, we have $u \in(\mathcal{E})$. Hence, $(\mathcal{E})_{Z} \cap \mathcal{A}=(\mathcal{E})$.

Let $\Gamma:=\mathbb{K}[E, Z]$. Then $\Lambda=\Gamma \oplus \Gamma X$ is a free $\Gamma$-module of rank 2 . Clearly, $X \Lambda=\Gamma E Z \oplus \Gamma X$ (since $\left.\mathcal{E}=E X-\frac{1}{2} X^{2}\right)$ and $Z^{i} \Lambda=Z^{i} \Gamma \oplus Z^{i} \Gamma X$ for all $i \in \mathbb{N}$. So, in the algebra (see Lemma 2)

$$
\overline{\mathcal{A}}:=\mathcal{A} /(\mathcal{E})=(\Gamma \oplus \Gamma X)\left[Y ; \delta_{1}\right]\left[H ; \delta_{2}\right]
$$

it is very easy to decide whether an element belongs to the left ideal $X \overline{\mathcal{A}}$ or the left ideal $Z^{i} \overline{\mathcal{A}}$. Clearly, $\bigcap_{i \geqslant 0} Z^{i} \overline{\mathcal{A}}=0$. So, for every nonzero element $a \in \overline{\mathcal{A}}$, there is a unique natural number $d=v_{Z}(a)$ such that $a \in Z^{d} \overline{\mathcal{A}} \backslash Z^{d+1} \overline{\mathcal{A}}$. The map $Z \cdot: \overline{\mathcal{A}} \rightarrow \overline{\mathcal{A}}, b \mapsto Z b$ is an injection. Therefore, $Z^{-v(a)} a \in \overline{\mathcal{A}}$ and $v_{Z}\left(Z^{-v_{Z}(a)} a\right)=0$. The element

$$
a_{\text {red }}:=Z^{-v_{Z}(a)} a
$$


is called the reduced form of $a$. Since $\mathcal{E}=E Z-\frac{1}{2} X^{2}$, we have the equality of ideals $(\mathcal{E}, Z)=\left(Z, X^{2}\right)$ in $\mathcal{A}$. By Lemma 2 ,

$$
\widetilde{\mathcal{A}}:=\mathcal{A} /(\mathcal{E}, Z)=\mathcal{A} /\left(Z, X^{2}\right) \simeq \frac{\mathbb{K}[E, X]}{X^{2} \mathbb{K}[E, X]}\left[Y ; \delta_{1}\right]\left[H ; \delta_{2}\right] .
$$

The element $X \in \widetilde{\mathcal{A}}$ is a normal element: $X Y=Y X$ and $X H=(H-1) X$ in $\widetilde{\mathcal{A}}$. Clearly, $X^{2}=0,(X)^{2}=\left(X^{2}\right)=0$ in $\widetilde{\mathcal{A}}$ and

$$
\widetilde{\mathcal{A}} /(X) \simeq \mathcal{A} /(X) \simeq \mathbb{K}[E]\left[Y ; \delta_{1}\right]\left[H ; \delta_{2}\right]
$$

is a domain. Therefore, the set of left zero divisors of $\widetilde{\mathcal{A}}$ is equal to the set of right zero divisors of $\widetilde{\mathcal{A}}$ and is equal to the ideal $(X)$ of $\widetilde{\mathcal{A}}$ since the left/right $\widetilde{A}$-module $(X)$ is isomorphic to $\widetilde{A}$. The set $\mathcal{C}_{\widetilde{\mathcal{A}}}$ of regular elements (i.e., nonzero divisors) of $\widetilde{\mathcal{A}}$ is equal to $\widetilde{\mathcal{A}} \backslash(X)$. The algebra $\widetilde{\mathcal{A}}$ is an epimorphic image of $\overline{\mathcal{A}}$.

The next lemma is used in the proof of Theorem 3.

Lemma 3. $\mathcal{H}^{2} \equiv \Phi Z \bmod (\mathcal{E})$ where $\Phi:=H^{2} Z+2 H X Y+2 E Y^{2}-X Y$. Proof. Using the defining relations of $\mathcal{A}$,

$$
\begin{aligned}
\mathcal{H}^{2} & =(H Z+X Y)^{2}=H^{2} Z^{2}+2 H X Y Z+(X Y)^{2} \\
& =H^{2} Z^{2}+2 H X Y Z+X^{2} Y^{2}-X Y Z \\
& =\left(H^{2} Z^{2}+2 H X Y Z+2 E Y^{2} Z-X Y Z\right)-2 E Y^{2} Z+X^{2} Y^{2} \\
& =\Phi Z-2 \mathcal{E} Y^{2}
\end{aligned}
$$

and the result follows.

The next theorem is a key result in finding explicit generators for prime ideals of the algebra $\mathcal{A}$. In general, as a rule, it is not possible to find explicit generators for a prime ideal of the form $\mathcal{A} \cap P$ where $P$ is a prime ideal of a localization $S^{-1} \mathcal{A}$ of $\mathcal{A}$.

Theorem 3. Let $f=a_{0}(Z)+a_{1}(Z) \mathcal{H}+a_{2}(Z) \mathcal{H}^{2}+\cdots+a_{n}(Z) \mathcal{H}^{n} \in$ $\mathbb{K}[Z, \mathcal{H}] \backslash(\mathbb{K} \cup \mathbb{K} Z)$ where all $a_{i}(Z) \in \mathbb{K}[Z]$ and $a_{0}(Z) \neq 0$. Then

1) $\mathcal{A} \cap(\mathcal{E}, f)_{Z}=(\mathcal{E}, f)$ iff $a_{0}(0) \neq 0$ iff $f \notin(Z, \mathcal{H})$ where $(Z, \mathcal{H})$ is the maximal ideal of the polynomial algebra $\mathbb{K}[Z, \mathcal{H}]$ generated by the elements $Z$ and $\mathcal{H}$.

2) Suppose, in addition, that $a_{0}(0)=0$. Let $d=v_{Z}(\bar{f}), \bar{f}_{\text {red }}=Z^{-d} \bar{f}$ and $\tilde{f}_{\text {red }}$ is the image of the element $\bar{f}_{\text {red }}$ under the epimorphism $\overline{\mathcal{A}} \rightarrow \widetilde{\mathcal{A}}$. 
(a) If $\tilde{f}_{\text {red }} \notin(X)$ then $\mathcal{A} \cap(\mathcal{E}, f)_{Z}=\left(\mathcal{E}, \bar{f}_{\text {red }}\right)$.

(b) If $\tilde{f}_{\text {red }} \in(X)$ then we have $\mathcal{A} \cap(\mathcal{E}, f)_{Z}=\left(\mathcal{E}, \bar{f}_{\text {red }}, Z^{-1} \bar{f}_{\text {red }} X\right)=$ $\left(\mathcal{E}, \bar{f}_{\text {red }}\right)+Z^{-1} \bar{f}_{\text {red }} X \mathcal{A}$.

Proof. 1. The equality $\mathcal{A} \cap(\mathcal{E}, f)_{Z}=(\mathcal{E}, f)$ holds iff the image $\tilde{f} \in \widetilde{\mathcal{A}}$ of the element $f$ under the epimorphism $\mathcal{A} \rightarrow \overline{\mathcal{A}} \rightarrow \widetilde{\mathcal{A}}$ is a nonzero divisor, i.e., $\tilde{f} \notin(X)$. By Lemma $3, \bar{f}=a_{0}(0)+a_{1}(0) X Y$ (since $\mathcal{H}=H Z+X Y$ ). So, $\tilde{f} \notin(X)$ iff $a_{0}(0) \neq 0$.

2. Clearly, $(\mathcal{E}, f)_{Z}=\left(\mathcal{E}, \bar{f}_{\text {red }}\right)_{Z}$ and $\tilde{f}_{\text {red }} \neq 0$ in $\widetilde{\mathcal{A}}$ since $v_{Z}(\bar{f})=0$.

(a) If $\tilde{f}_{\text {red }} \notin(X)$ then $\mathcal{A} \cap(\mathcal{E}, f)_{Z}=\mathcal{A} \cap\left(\mathcal{E}, \bar{f}_{\text {red }}\right)_{Z}=\left(\mathcal{E}, \bar{f}_{\text {red }}\right)$, by statement 1 .

(b) If $\tilde{f}_{\text {red }} \in(X)$ then $\tilde{f}_{\text {red }}=\alpha X$ for some nonzero element $\alpha \in \mathcal{A} /(X)$ since

$$
\widetilde{\mathcal{A}} X=\mathcal{A} /(X) \cdot X
$$

(as $(X)^{2}=0$ in $\left.\widetilde{\mathcal{A}}\right)$. The element $\alpha$ is unique since the left $\mathcal{A} /(X)$-module $\mathcal{A} /(X) \cdot X$ is free of rank 1. If $a \in \mathcal{A} \cap\left(\mathcal{E}, \bar{f}_{\text {red }}\right)_{Z} \backslash\left(\mathcal{E}, \bar{f}_{\text {red }}\right)$ then the element $\bar{a}=a+(\mathcal{E}) \in \overline{\mathcal{A}}$ is a product $\bar{a}=Z^{-i} \bar{f}_{\text {red }} b$ for some $b \in \overline{\mathcal{A}}$ with $v_{Z}(b)=0$ where $i=v_{Z}\left(\bar{f}_{\text {red }} b\right) \geqslant 1$. We may assume that $v_{Z}(\bar{a})=0$. The image $\tilde{b}$ of the element $b$ in $\widetilde{\mathcal{A}}$ is a nonzero one since $v_{Z}(b)=0$. Since $\tilde{f}_{\text {red }} \tilde{b}=0$ in $\widetilde{\mathcal{A}}$ and $\tilde{f}_{\text {red }} \neq 0$, the element $\tilde{b} \in \widetilde{\mathcal{A}}$ is a zero divisor, i.e., $\tilde{b} \in X \widetilde{\mathcal{A}}$. Therefore, $\tilde{b}=X \beta$ for a nonzero element $\beta \in \mathcal{A} /(X)$ since $X \cdot \widetilde{\mathcal{A}}=X \cdot \mathcal{A} /(X)$ (as $(X)^{2}=0$ in $\left.\widetilde{\mathcal{A}}\right)$. Now,

$$
Z^{i} \bar{a}=\bar{f}_{\text {red }} b \equiv \tilde{f}_{\text {red }} \tilde{b} \equiv \alpha X \cdot X \beta \equiv \alpha 2 E Z \beta \equiv 0 \bmod (Z \bar{A}) .
$$

Since the image of the element $\alpha 2 E \beta$ in the domain $\mathcal{A} /(X)$ is a nonzero one, we must have $i=1$ (since $v_{Z}(\bar{a})=0$ and $v_{Z}\left(Z^{i} a\right)=i+v_{Z}(a)=i$ ). Therefore,

$$
\bar{a}=Z^{-1} \bar{f}_{\text {red }} b \in Z^{-1} \bar{f}_{\text {red }}(X \overline{\mathcal{A}}+Z \overline{\mathcal{A}}) \subseteq Z^{-1} \bar{f}_{\text {red }} X \overline{\mathcal{A}}+\bar{f}_{\text {red }} \overline{\mathcal{A}} .
$$

This means that $\mathcal{A} \cap(\mathcal{E}, f)_{Z}=\mathcal{A} \cap\left(\mathcal{E}, \bar{f}_{\text {red }}\right)_{Z}=\left(\mathcal{E}, \bar{f}_{\text {red }}\right)+Z^{-1} \bar{f}_{\text {red }} X \overline{\mathcal{A}}=$ $\left(\mathcal{E}, \bar{f}_{\text {red }}, Z^{-1} \bar{f}_{\text {red }} X\right)$.

Example. Let $f=\mathcal{H}$. Then $\mathfrak{p}_{0}=(f) \in \mathcal{P}_{0}$ and $\mathcal{A} \cap\left(\mathcal{E}, \mathfrak{p}_{0}\right)_{Z}=$ $(\mathcal{E}, \mathcal{H}, H X+2 E Y)$ : Clearly, $\mathfrak{p}_{0} \in \mathcal{P}_{0}$. Then $\bar{f}_{\text {red }}=\bar{f}=\mathcal{H}=H Z+X Y$ and $Z^{-1} \bar{f}_{\text {red }} X=Z^{-1}\left(Z H X+X^{2} Y\right)=H X+Z^{-1} 2 E Z Y=H X+2 E Y$. Now, the result follows from Theorem 1.

Example. Let $f=Z+Z \mathcal{H}+\mathcal{H}^{2}$. Then $\mathfrak{p}_{0}=(f) \in \mathcal{P}_{0}$ and $\mathcal{A} \cap\left(\mathcal{E}, \mathfrak{p}_{0}\right)_{Z}=$ $\left(\mathcal{E}, 1+\mathcal{H}+2 E Y^{2}\right):$ Clearly, $\mathfrak{p}_{0} \in \mathcal{P}_{0}$ and $\bar{f}=Z+Z \mathcal{H}+X^{2} Y^{2}=Z(1+$ $\left.\mathcal{H}+2 E Y^{2}\right)$. Hence, $\bar{f}_{\text {red }}=1+\mathcal{H}+2 E Y^{2}$. Now, the result follows from Theorem 1. 
Example. Let $f=Z^{2}+Z \mathcal{H}+\mathcal{H}^{2}$. Then $\mathfrak{p}_{0}=(f) \in \mathcal{P}_{0}$ and $\mathcal{A} \cap\left(\mathcal{E}, \mathfrak{p}_{0}\right)_{Z}=$ $\left(\mathcal{E}, Z+\mathcal{H}+2 E Y^{2}\right):$ Clearly, $\mathfrak{p}_{0} \in \mathcal{P}_{0}$ and $\bar{f}=Z^{2}+Z \mathcal{H}+X^{2} Y^{2}=Z(Z+$ $\left.\mathcal{H}+2 E Y^{2}\right)$. Hence, $\bar{f}_{\text {red }}=Z+\mathcal{H}+2 E Y^{2}$. Since $\tilde{f}_{\text {red }}=X Y+2 E Y^{2} \notin(X)$, we have the result, by Theorem 1 .

Example. Let $f=Z^{2}+\mathcal{H}^{3}$. Then $\mathfrak{p}_{0}=(f) \in \mathcal{P}_{0}$ and $\mathcal{A} \cap\left(\mathcal{E}, \mathfrak{p}_{0}\right)_{Z}=$ $\left(\mathcal{E}, Z+2 E X Y, 1+4 E^{2} Y\right):$ Clearly, $\mathfrak{p}_{0} \in \mathcal{P}_{0}$ and $\bar{f}=Z^{2}+X^{3} Y^{3}=$ $Z^{2}+2 Z E X Y$. Then $\bar{f}_{\text {red }}=Z+2 E X Y$. Since $\tilde{f}_{\text {red }}=2 E X Y \in(X)$, $Z^{-1} \bar{f}_{\text {red }} X=1+Z^{-1} 2 E X^{2} Y=1+Z^{-1} 2 E \cdot 2 Z E Y=1+4 E^{2} Y$. Now, the result follows from Theorem 1 .

Proof of Theorem 1. By (5) and (6), the set $\operatorname{Spec}(\mathcal{A})$ is a disjoint union of the sets $\operatorname{Spec}(\mathcal{A} /(Z)), \operatorname{Spec}\left(\mathcal{A}_{Z} /(\mathcal{E})_{Z}\right)$ and $\operatorname{Spec}\left(\mathcal{A}_{Z, \mathcal{E}}\right)$. Recall that we view these sets as subsets of $\operatorname{Spec}(\mathcal{A})$. We have seen above that $\operatorname{Spec}(\mathcal{A} /(Z))$ contains precisely the prime ideals over $(Z)$ in the diagram (2).

(i) The set (as a subset of $\operatorname{Spec}(\mathcal{A})) \operatorname{Spec}\left(\mathcal{A}_{Z, \mathcal{E}}\right)$ is equal to $\{0\} \sqcup$ $\{(\mathfrak{q}) \mid \mathfrak{q} \in \mathcal{Q}\}$ where $\mathcal{Q}=\operatorname{Max}(\mathbb{K}[Z]) \backslash\{(Z)\}$ : By (8), each nonzero element of $\operatorname{Spec}\left(\mathcal{A}_{Z, \mathcal{E}}\right)$ is equal to $\mathcal{A} \cap(\mathfrak{q})_{Z, \mathcal{E}}$ for some $\mathfrak{q} \in \mathcal{Q}$. We have to show that $\mathcal{A} \cap(\mathfrak{q})_{Z, \mathcal{E}}=\mathfrak{q} \mathcal{A}$. The ideal $(\mathfrak{q})=\mathfrak{q} \mathcal{A}$ is a completely prime ideal of $\mathcal{A}$ since, by (1),

$$
\mathcal{A} / \mathcal{A q} \simeq \mathcal{A}_{Z} / \mathcal{A}_{Z} \mathfrak{q} \simeq \mathbb{K}\left[Z^{ \pm 1}\right] /(\mathfrak{q})_{Z} \otimes \mathbb{K}\left[H^{\prime}\right][\mathcal{E} ; \sigma] \otimes A_{1},
$$

a domain. Hence, $(\mathfrak{q})=\mathcal{A} \cap(\mathfrak{q})_{Z}$. By $(10), \mathcal{A} \cap(\mathfrak{q})_{Z, \mathcal{E}}=\mathcal{A} \cap(\mathfrak{q})_{Z}$, and so $(\mathfrak{q})=\mathcal{A} \cap(\mathfrak{q})_{Z, \mathcal{E}}$, as required.

Let us describe the set $\operatorname{Spec}\left(\mathcal{A}_{Z} /(\mathcal{E})_{Z}\right)$. By $(7)$, we see that the set $\operatorname{Spec}\left(\mathcal{A}_{Z} /(\mathcal{E})_{Z}\right)$ (as a subset of $\left.\operatorname{Spec}(\mathcal{A})\right)$ consists of the elements: $(\mathcal{E})=$ $\mathcal{A} \cap(\mathcal{E})_{Z}$ (by Lemma 2$), \mathcal{A} \cap \mathcal{A}_{Z}(\mathcal{E}, \mathfrak{m})$ where $\mathfrak{m} \in \mathcal{M}$, and $\mathcal{A} \cap \mathcal{A}_{Z}(\mathcal{E}, \mathfrak{p})$ where $\mathfrak{p}$ is a prime, height 1 ideal of $\mathbb{K}[Z, \mathcal{H}]$ such that $\mathfrak{p} \neq(Z)$. The last set of prime ideals is equal to the set $\left\{\mathcal{A} \cap\left(\mathcal{E}, \mathfrak{p}_{0}\right)_{Z}, \mathcal{A} \cap\left(\mathcal{E}, \mathfrak{p}_{1}\right)_{Z} \mid \mathfrak{p}_{0} \in\right.$ $\left.\mathcal{P}_{0}, \mathfrak{p}_{1} \in \mathcal{P}_{1}\right\}$.

(ii) For all $\mathfrak{m} \in \mathcal{M},(\mathcal{E}, \mathfrak{m})=\mathcal{A} \cap(\mathcal{E}, \mathfrak{m})_{Z}$ is a maximal (completely prime) ideal of $\mathcal{A}$ : The element $Z$ is a unit of the field $F_{\mathfrak{m}}:=\mathbb{K}[Z, \mathcal{H}] /(\mathfrak{m})$. Therefore, by (1),

$$
\mathcal{A} /(\mathcal{E}, \mathfrak{m}) \simeq \mathcal{A}_{Z} /(\mathcal{E}, \mathfrak{m})_{Z} \simeq F_{\mathfrak{m}} \otimes A_{1},
$$

a simple domain. Hence, the ideal $(\mathcal{E}, \mathfrak{m})=\mathcal{A} \cap(\mathcal{E}, \mathfrak{m})_{Z}$ is a maximal (completely prime) ideal of $\mathcal{A}$. 
(iii) For every $\mathfrak{q} \in \mathcal{Q}$, the ideal $(\mathcal{E}, \mathfrak{q})=\mathcal{A} \cap(\mathcal{E}, \mathfrak{q})_{Z}$ of $\mathcal{A}$ is completely prime: The element $Z$ is a unit of the field $L_{\mathfrak{q}}:=\mathbb{K}[Z] / \mathfrak{q}$. By (1),

$$
\mathcal{A} /(\mathcal{E}, \mathfrak{q}) \simeq \mathcal{A}_{Z} /(\mathcal{E}, \mathfrak{q})_{Z} \simeq L_{\mathfrak{q}} \otimes \mathbb{K}\left[H^{\prime}\right] \otimes A_{1}
$$

a domain. Hence, the ideal $(\mathcal{E}, \mathfrak{q})=\mathcal{A} \cap(\mathcal{E}, \mathfrak{q})_{Z}$ is a completely prime ideal.

(iv) For all $\mathfrak{p}_{1} \in \mathcal{P}_{1}, \mathcal{A} \cap\left(\mathcal{E}, \mathfrak{p}_{1}\right)_{Z}=\left(\mathcal{E}, \mathfrak{p}_{1}\right)$ : This follows form Theorem 3.(1).

(v) Let $\mathfrak{p}_{0} \in \mathcal{P}_{0}$, then $\left(\mathfrak{p}_{0}\right)=(f)$ for some irreducible polynomial $f \in \mathbb{K}[Z, \mathcal{H}] \backslash(\mathbb{K} \cup \mathbb{K} Z)$ as in Theorem 3 with $a_{0}(0)=0$. Then (3) holds: This follow from Theorem 3.(2).

So, we have proved that the diagram (2) contains precisely all the prime ideals of the algebra $\mathcal{A}$.

(vi) All the containments between primes are given in (2): Repeating twice Proposition 1.(c), we see that possible inclusions between prime ideals $P_{1}, P_{2}$ and $P_{3}$ of the three sets $\operatorname{Spec}(\mathcal{A} /(Z))$, Spec $\left(\mathcal{A}_{Z} /(\mathcal{E})_{Z}\right)$ and $\operatorname{Spec}\left(\mathcal{A}_{Z, \mathcal{E}}\right)$, respectively, can only possibly be $P_{1} \supset P_{2}, P_{1} \supset P_{3}$ or $P_{2} \supset P_{3}$. It is easy to see that we have the inclusions between prime ideals as in the diagram (2) (see also below for details). Clearly, the only possible inclusions $P_{1} \supset P_{3}$ and $P_{2} \supset P_{3}$ are as in the diagram (2).

It remains to sort out inclusions of the type $P_{1} \supset P_{2}$. By the statement (ii), $P_{2}$ is not equal to the maximal ideal $(\mathcal{E}, \mathfrak{m})$ where $\mathfrak{m} \in \mathcal{M}$. By the very definition the elements $Z, \mathcal{E}$ and $\mathcal{H}$ belong to the ideal $(X)$. Hence, $(X) \supseteq(\mathcal{E})$. It remains to consider the case where $P_{2}$ is of type $\mathcal{A} \cap(\mathcal{E}, \mathfrak{p})_{Z}$ where $\mathfrak{p} \in \mathcal{P}_{0} \cup \mathcal{P}_{1}$. Clearly, $(X) \supseteq \mathcal{A} \cap\left(\mathcal{E}, \mathfrak{p}_{0}\right)_{Z}$ for all $\mathfrak{p}_{0} \in \mathcal{P}_{0}$ (since $Z, \mathcal{E}, \mathcal{H} \in(X))$ and $P_{1} \nsupseteq \mathcal{A} \cap\left(\mathcal{E}, \mathfrak{p}_{1}\right)$ for all $P_{1}=(Y, E, \mathfrak{p})$ where $\mathfrak{p} \in \operatorname{Max}(\mathbb{K}[H])$ and all $\mathfrak{p}_{1} \in \mathcal{P}_{1}$ since $P_{1} \supseteq(X)$ and $Z, \mathcal{H} \in(X)$ (and so the maximal ideal of $\mathbb{K}[Z, \mathcal{H}]$ generated by the elements $Z$ and $\mathcal{H}$ is contained in $(X)$ and, therefore, in all $P_{1}=(Y, E, \mathfrak{p})$; clearly, the ideal of $\mathbb{K}[Z, \mathcal{H}]$ generated by $Z, \mathcal{H}$ and $P_{1}$ is $\left.(1)\right)$. So, all inclusions between primes are as in (2).

As a corollary of Theorem 1 we obtain a classification of maximal ideals of $\mathcal{A}$.

Corollary 1. The set of maximal ideals of $\mathcal{A}$ is equal to $\left\{\left(X, \mathfrak{q}^{\prime}\right) \mid \mathfrak{q}^{\prime} \in\right.$ $\left.\mathcal{Q}^{\prime}\right\} \sqcup\{(Y, E, \mathfrak{p}) \mid \mathfrak{p} \in \operatorname{Max}(\mathbb{K}[H])\} \sqcup\{(\mathcal{E}, \mathfrak{m}) \mid \mathfrak{m} \in \mathcal{M}\}$ where $\mathcal{Q}^{\prime}$ and $\mathcal{M}$ are defined in Theorem 1

Proof. The corollary follows from Theorem 1. 
Corollary 2. Not every nonzero prime ideal of the algebra $\mathcal{A}$ meets the centre.

Proof. The statement follows from (2).

The set $\operatorname{Prim}(\mathcal{A})$ of primitive ideals of the algebra $\mathcal{A}$. An ideal of a ring $R$ is called a primitive ideal if it is the annihilator of a simple left $R$-module. The set of primitive ideals of $R$ is denoted by $\operatorname{Prim}(R)$. A prime ideal $P$ of a ring $R$ is said to be locally closed if the set $\{P\}$ is locally closed in the topological space $\operatorname{Spec}(R)$ where $\operatorname{Spec}(R)$ is equipped with Zariski-Jacobson topology [8, II.1.1]. A prime ideal $P$ of a Noetherian $\mathbb{K}$-algebra $R$ is said to be rational if the field $Z(\operatorname{Frac}(R / P))$ is algebraic over $\mathbb{K}$ where $\operatorname{Frac}(R / P)$ is the left (right) quotient ring of the Noetherian prime algebra $R / P$. We say that the Dixmier-Moeglin equivalence holds for a Noetherian $\mathbb{K}$-algebra $A$ if for each prime ideal $P$ of $A$ we have the following equivalences:

$P$ is locally closed $\Longleftrightarrow P$ is primitive $\Longleftrightarrow P$ is rational.

The next corollary describes the set of primitive ideals $\operatorname{Prim}(\mathcal{A})$ of the algebra $\mathcal{A}$.

Corollary 3. $\operatorname{Prim}(\mathcal{A})=\operatorname{Max}(\mathcal{A}) \sqcup\{(Y),(E),(Z)\} \sqcup\{(\mathfrak{q}) \mid \mathfrak{q} \in \mathcal{Q}\}$.

Proof. Since $\mathcal{A}$ is a universal enveloping algebra of a finite dimensional Lie algebra it satisfies the Dixmier-Moeglin equivalence. By [8, Lemma II.7.7], a prime ideal $P$ in a ring $R$ is locally closed iff the intersection of all prime ideals properly containing $P$ is also an ideal properly containing $P$. Clearly, all the maxial ideals are primitive ideals. By (2), the set of locally closed prime ideals is $\operatorname{Max}(\mathcal{A}) \sqcup\{(Y),(E),(Z)\} \sqcup\{(\mathfrak{q}) \mid \mathfrak{q} \in \mathcal{Q}\}$. Then the corollary follows from the Dixmier-Moeglin equivalence for $\mathcal{A}$.

The semicentre of $\mathcal{A}$. Recall that $\mathcal{A}=U(\mathfrak{a})$ is the enveloping algebra of the solvable Lie algebra a. For each $\lambda \in \operatorname{Hom}_{\mathbb{K}}(\mathfrak{a}, \mathbb{K})$, let $\mathcal{A}_{\lambda}:=\{a \in$ $\left.\mathcal{A} \mid \operatorname{ad}_{x}(a)=\lambda(x) a, \forall x \in \mathfrak{a}\right\}$. Any nonzero element $a \in \mathcal{A}_{\lambda}$ is called a semi-invariant with weight $\lambda$. The sum of the $\mathcal{A}_{\lambda}$ is direct and is denoted by $S z(\mathcal{A})$ which contains the centre of $\mathcal{A} . S z(\mathcal{A})$ is called the semicentre of $\mathcal{A}$, which is a commutative domain, (see [15, Corollary 4.6]).

Proposition 2. $S z(\mathcal{A})=\mathbb{K}[Z, \mathcal{E}]$. 
Proof. It is clear that $Z$ and $\mathcal{E}$ are semi-invariants of the algebra $\mathcal{A}$. Since $Z(\operatorname{Frac}(\mathcal{A}))=\mathbb{K}(Z)$, by [10, Proposition 16.(c)], $S z(\mathcal{A})$ is a polynomial algebra over $\mathbb{K}$. Since ind $(\mathfrak{a})=1$ where ind $(\mathfrak{a})$ is the index of the Lie algebra $\mathfrak{a}$ and $\operatorname{deg}(Z)+\operatorname{deg}(\mathcal{E})=3=\frac{1}{2}(\operatorname{dim} \mathfrak{a}+\operatorname{ind}(\mathfrak{a}))$, by [10, Lemma 17] and [16, Theorem 1.1], we have $S z(\mathcal{A})=\mathbb{K}[Z, \mathcal{E}]$, as required.

The factor algebra $\mathcal{A} /(Z-\lambda)$. For $\lambda \in \mathbb{K}^{*}$, let $\mathcal{A}(\lambda):=\mathcal{A} /(Z-\lambda)$.

Lemma 4. $\mathcal{A}(\lambda) \simeq \mathbb{K}\left[H_{\lambda}\right]\left[E_{\lambda} ; \sigma\right] \otimes A_{1}$ where $H_{\lambda}=H+\lambda^{-1} X Y-\frac{1}{2}$, $E_{\lambda}=E-\frac{1}{2} \lambda^{-1} X^{2}, \sigma$ is the automorphism of the algebra $\mathbb{K}\left[H_{\lambda}\right]$ defined by $\sigma\left(H_{\lambda}\right)=H_{\lambda}-2$ and $A_{1}=\mathbb{K}\left\langle\lambda^{-1} X, Y\right\rangle$ is the first Weyl algebra.

Proof. Notice that the element $Z+(Z-\lambda)=\lambda+(Z-\lambda) \in \mathcal{A}(\lambda)$ is an invertible element, so $\mathcal{A}(\lambda) \simeq \mathcal{A}_{Z} /(Z-\lambda)_{Z}$. Then the lemma follows from (1).

Whittaker $\mathcal{A}(\boldsymbol{\lambda})$-modules. Let $U^{+}=\mathbb{K}[H][E ; \sigma]$ where $\sigma(H)=$ $H-2$. Then $U^{+}$is the 'positive' part of the enveloping algebra $U=U\left(\mathfrak{s l}_{2}\right)$.

Lemma 5. For $\alpha \in \mathbb{K}$, let $M=U^{+} / U^{+}(E-\alpha)$ be the left $U^{+}{ }_{\text {-module. }}$ Then $M$ is a simple module iff $\alpha \neq 0$.

For $\mu, \delta \in \mathbb{K}$, let

$$
W(\mu, \delta):=\mathcal{A}(\lambda) / \mathcal{A}(\lambda)(E-\mu, X-\delta),
$$

a left $\mathcal{A}(\lambda)$-module. We call $W(\mu, \delta)$ the Whittaker $\mathcal{A}(\lambda)$-module of type $(\mu, \delta)$. The following proposition is a simplicity criterion of the module $W(\mu, \delta)$.

Proposition 3. $W(\mu, \delta)$ is a simple $\mathcal{A}(\lambda)$-module iff $\delta^{2}-2 \lambda \mu \neq 0$.

Proof. Let $U^{\prime+}=\mathbb{K}\left[H_{\lambda}\right]\left[E_{\lambda} ; \sigma\right]$ where $\sigma\left(H_{\lambda}\right)=H_{\lambda}-2$. By Lemma 4, $\mathcal{A}(\lambda)=U^{\prime+} \otimes A_{1}$. Then

$$
\begin{aligned}
W(\mu, \delta) & =\mathcal{A}(\lambda) / \mathcal{A}(\lambda)\left(E_{\lambda}+\frac{1}{2} \lambda^{-1} X^{2}-\mu, X-\delta\right) \\
& =\mathcal{A}(\lambda) / \mathcal{A}(\lambda)\left(E_{\lambda}+\frac{1}{2} \lambda^{-1} \delta^{2}-\mu, X-\delta\right) \\
& \simeq U^{\prime+} / U^{\prime+}\left(E_{\lambda}+\frac{1}{2} \lambda^{-1} \delta^{2}-\mu\right) \otimes A_{1} / A_{1}(X-\delta) .
\end{aligned}
$$

Let $W^{\prime}:=U^{\prime+} / U^{\prime+}\left(E_{\lambda}+\frac{1}{2} \lambda^{-1} \delta^{2}-\mu\right)$. Notice that $A_{1} / A_{1}(X-\delta)$ is a simple $A_{1}$-module with $\operatorname{End}\left(A_{1} / A_{1}(X-\delta)\right)=\mathbb{K}$. Then $W(\mu, \delta)$ is a simple $\mathcal{A}(\lambda)$-module iff $W^{\prime}$ is simple $U^{\prime+}$-module iff $\frac{1}{2} \lambda^{-1} \delta^{2}-\mu \neq 0$, i.e. $\delta^{2}-2 \lambda \mu \neq 0$, by Lemma 5 . 


\section{Prime ideals of the universal enveloping algebra $U\left(\mathfrak{g l}_{2}\right)$}

In this section, we describe the prime, maximal, completely prime and primitive ideals of the enveloping algebra $U\left(\mathfrak{g l}_{2}\right)$ (Theorem 2, Corollary 4 and Corollary 5). Recall that the Lie algebra $\mathfrak{g l}_{2}$ is the direct product of the Lie algebra $\mathfrak{s l}_{2}$ and a 1 -dimensional Lie algebra $\mathbb{K} Z$. So, $Z$ is a central element of the algebra $\mathcal{U}:=U\left(\mathfrak{g l}_{2}\right)$. Clearly, $\mathcal{U}=U\left(\mathfrak{s l}_{2}\right) \otimes \mathbb{K}[Z]$ is a tensor product of algebras. Hence, $Z(\mathcal{U})=Z\left(U\left(\mathfrak{s l}_{2}\right)\right) \otimes \mathbb{K}[Z]=\mathbb{K}[\Delta, Z]$ where $\Delta=4 F E+H^{2}+2 H$ is the Casimire element of $U\left(\mathfrak{s l}_{2}\right)$. The algebra

$$
\mathcal{U}=\mathbb{K}[Z, \Delta, H]\left[E, F ; \sigma, a=\frac{1}{4}\left(\Delta-H^{2}-2 H\right)\right]
$$

is a generalized Weyl algebra (GWA) where $\sigma(Z)=Z, \sigma(\Delta)=\Delta$ and $\sigma(H)=H-2$, see [1,2] for the definition of GWAs and classification of ideals of GWAs. Clearly, $\mathcal{U}$ is a free module over its centre $\mathbb{K}[Z, \Delta]$.

Proof of Theorem 2. Let $\mathcal{Z}=\mathbb{K}[Z, \Delta]$.

(i) For each prime ideal $\mathfrak{p}$ of $\mathcal{Z}$, the ideal $(\mathfrak{p})=\mathfrak{p} \mathcal{U}$ of $\mathcal{U}$ is a completely prime ideal: The statement (i) follows from the fact that the factor algebra

$$
\mathcal{U} / \mathfrak{p} \mathcal{U} \simeq \mathcal{Z} / \mathfrak{p}[H][E, F ; \sigma, a]
$$

is a GWA which is a domain.

(ii) For all non-zero prime ideals $P$ of $\mathcal{U}, P \cap \mathcal{Z} \neq 0$ : The localization of the algebra $\mathcal{U}$ at the set $\mathcal{Z} \backslash\{0\}$ is the simple generalized Weyl algebra $\mathbb{K}(Z, \Delta)[H][E, F ; \sigma, a]$, since the element $a$ is irreducible in the Dedekind domain $\mathbb{K}(Z, \Delta)[H]$ and the group $\langle\sigma\rangle$ acts freely on $\operatorname{Max}(\mathbb{K}(Z, \Delta)[H])$, see the description of all the ideals in [2]. Now, the statement (ii) is obvious.

Till the end of the proof of the theorem $P$ is a nonzero prime ideal of $\mathcal{U}$. Then $P^{\prime}:=\mathcal{Z} \cap P \in \operatorname{Spec}(\mathcal{Z})$. Let $T:=\mathbb{K}[Z] \backslash\{0\}$. Then

$$
T^{-1} \mathcal{U}=\mathbb{K}(Z) \otimes U=\mathbb{K}(Z)[\Delta, H][E, F ; \sigma, a]
$$

is the universal enveloping algebra of $\mathfrak{s l}_{2}$ over the field $\mathbb{K}(Z)$. Then

$$
\operatorname{Spec}(\mathcal{U})=\bigsqcup_{\mathfrak{q} \in \operatorname{Max}(\mathbb{K}[Z])} \operatorname{Spec}(\mathcal{U} / \mathfrak{q} \mathcal{U}) \sqcup \operatorname{Spec}\left(T^{-1} \mathcal{U}\right)
$$

Let $L_{\mathfrak{q}}:=\mathbb{K}[Z] / \mathfrak{q}$, a finite field extension of $\mathbb{K}$. For every $\mathfrak{q} \in \operatorname{Max}(\mathbb{K}[Z])$, the algebra

$$
\mathcal{U} / \mathfrak{q} \mathcal{U}=L_{\mathfrak{q}} \otimes U=L_{\mathfrak{q}}[\Delta, H][E, F ; \sigma, a]
$$

is a GWA. Using the classification of the ideals of the algebra $U$ in [2], 
(a) the set $\operatorname{Spec}(\mathcal{U} / \mathfrak{q} \mathcal{U})$, as a part of $\operatorname{Spec}(\mathcal{U})$, is identified with the set

$$
\left\{(\mathfrak{q}),\left(\Delta-\lambda_{n}, \mathfrak{q}\right),\left(I_{n}, \mathfrak{q}\right),(\mathfrak{m}) \mid n \in \mathbb{N}_{+}, \mathfrak{m} \in \operatorname{Max}(\mathcal{Z}) \text { and } \mathfrak{q} \subseteq \mathfrak{m}\right\},
$$

where $n \in \mathbb{N}_{+}, \lambda_{n}:=n^{2}-1$, and $I_{n}$ is the annihilator of the simple $n$-dimensional $U\left(\mathfrak{s l}_{2}\right)$-module,

(b) the set $\operatorname{Spec}\left(T^{-1} \mathcal{U}\right)$, as a part of $\operatorname{Spec}(\mathcal{U})$, is identified with the set

$$
\left\{0,\left(\Delta-\lambda_{n}\right),\left(I_{n}\right),(\mathfrak{p}),(\mathfrak{m}) \mid n \in \mathbb{N}_{+}, \mathfrak{p} \in \mathbb{H}_{1} \text { and } \mathfrak{m} \in \mathbb{H}_{2}\right\}
$$

Hence, the set of prime ideals is as in (4). The inclusions in (4) are obvious.

The next corollary classifies all the maximal and completely prime ideals of $\mathcal{U}$.

Corollary 4. 1) $\operatorname{Max}(\mathcal{U})=\left\{\left(I_{n}, \mathfrak{q}\right) \mid n \in \mathbb{N}_{+}, \mathfrak{q} \in \operatorname{Max}(\mathbb{K}[Z])\right\}$ $\left\{(\mathfrak{m}) \mid \mathfrak{m} \in \mathbb{H}_{2}\right\}$ where $\mathbb{H}_{2}=\operatorname{Max}(\mathbb{K}[Z, \Delta]) \backslash\left\{\left(\Delta-\lambda_{n}, \mathfrak{q}\right) \mid n \in\right.$ $\left.\mathbb{N}_{+}, \mathfrak{q} \in \operatorname{Max}(\mathbb{K}[Z])\right\}$.

2) The set of completely prime ideals of $\mathcal{U}$ is equal to $\operatorname{Spec}(\mathcal{U}) \backslash$ $\left\{\left(I_{n}\right),\left(I_{n}, \mathfrak{q}\right) \mid n \geqslant 2 ; \mathfrak{q} \in \operatorname{Max}(\mathbb{K}[Z])\right\}$.

Proof. 1. Statement 1 follows from (4).

2. For all $n \in \mathbb{N}_{+}$and $\mathfrak{q} \in \operatorname{Max}(\mathbb{K}[Z]), \mathcal{U} /\left(I_{n}\right)=\mathbb{K}[Z] \otimes U / I_{n} \simeq$ $\mathbb{K}[Z] \otimes M_{n}(\mathbb{K})$ and

$$
\mathcal{U} /\left(I_{n}, \mathfrak{q}\right) \simeq \mathbb{K}[Z] / \mathfrak{q} \otimes U / I_{n} \simeq \mathbb{K}[Z] / \mathfrak{q} \otimes M_{n}(\mathbb{K}) .
$$

For all $P \in \operatorname{Spec}(\mathcal{U}) \backslash\left\{\left(I_{n}\right),\left(I_{n}, \mathfrak{q}\right) \mid n \geqslant 2, \mathfrak{q} \in \operatorname{Max}(\mathbb{K}[Z])\right\}, \mathcal{U} / P \simeq$ $\mathcal{Z} / P^{\prime}[H][E, F ; \sigma, a]$ is a GWA which is a domain. Now statement 2 is obvious.

The next corollary describes the set of primitive ideals of $\mathcal{U}$.

Corollary 5. The set of primitive ideals of $\mathcal{U}$ is equal to $\operatorname{Max}(\mathcal{U})$ $\left\{\left(\Delta-\lambda_{n}, \mathfrak{q}\right) \mid n \in \mathbb{N}_{+}, \mathfrak{q} \in \operatorname{Max}(\mathbb{K}[Z])\right\}$.

Proof. The maximal ideals are prime. For all $n \in \mathbb{N}_{+}$and $\mathfrak{q} \in \operatorname{Max}(\mathbb{K}[Z])$, the factor algebra $\mathcal{U} /\left(\Delta-\lambda_{n}, \mathfrak{q}\right) \simeq \mathbb{K}[Z] / \mathfrak{q}[H][E, F ; \sigma, a]$ is a GWA for which zero ideal is primitive. Let $P$ be a prime ideal of $\mathcal{U}$ which does not belong to the union in the corollary, then the centre of the algebra $\mathcal{U} / P$ contains a transcendental element over $\mathbb{K}$. Hence, the ideal $P$ cannot be primitive. 


\section{Acknowledgements}

This paper was started when T. Lu was a postgraduate student at the School of Mathematics and Statistics of the University of Sheffield, UK (2012-2016) and finished when V. Bavula visited the School of Mathematical Sciences of the Huaqiao University, China (March-April 2018 and April 2019). The hospitality of both institutions is greatly appreciated.

\section{References}

[1] V. V. Bavula, Description of two-sided ideals in a class of noncommutative rings. I, Ukrainian Math. J., 45 (1993) no. 2, 223-234.

[2] V. V. Bavula, Description of two-sided ideals in a class of noncommutative rings. II, Ukrainian Math. J., 45 (1993) no. 3, 329-334.

[3] V. V. Bavula and T. Lu, The prime spectrum and simple modules over the quantum spatial ageing algebra, Algebr. Represent. Theory, 19 (2016), 1109-1133.

[4] V. V. Bavula and T. Lu, Classification of simple weight modules over the Schrödinger algebra, Canad. Math. Bull., 61 (2018), no. 1, 16-39.

[5] V. V. Bavula and T. Lu, The universal enveloping algebra $U\left(\mathfrak{s l}_{2} \ltimes V_{2}\right)$, its prime spectrum and a classification of its simple weight modules, J. Lie Theory, 28 (2018), no. $2,525-560$.

[6] V. V. Bavula and T. Lu, The universal enveloping algebra of the Schrödinger algebra and its prime spectrum, Canad. Math. Bull., 61 (2018), no. 4, 688-703.

[7] V. V. Bavula and T. Lu, The prime ideals and simple modules of the universal enveloping algebra $U\left(\mathfrak{b} \ltimes V_{2}\right)$, Glasg. Math. J., 62 (2020), no. S1, S77-S98

[8] K. A. Brown, K. R. Goodearl, Lectures on Algebraic Quantum Groups, Advanced Course in Math. CRM Barcelona, vol. 2. Birkhauser, Basel 2002.

[9] Y. Cai, Y. Cheng and R. Shen, Quasi-Whittaker modules for the Schrödinger algebra, Lin. Alg. Appl., 463 (2014) 16-32.

[10] L. Delvaux, E. Nauwelaerts, A. I. Ooms and P. Wauters, Primitive localizations of an enveloping algebra, J. Algebra, 130 (1990) 311-327.

[11] J. Dixmier, Enveloping Algebras, Graduate Studies in Mathematics, Vol. 11, 1996.

[12] B. Dubsky, Classification of simple weight modules with finite-dimensional weight spaces over the Schrödinger algebra, Lin. Alg. Appl., 443 (2014) 204-214.

[13] B. Dubsky, R. Lü, V. Mazorchuk, K. Zhao, Category $\mathcal{O}$ for the Schrödinger algebra, Lin. Alg. Appl., 460 (2014), 17-50.

[14] V. Dobrev, H.-D. Doebner, C. Mrugalla, Lowest weight representations of the Schrödinger algebra and generalized heat/Schrödinger equations, Rep. Math. Phys., 39 (1997), 201-218.

[15] J. C. McConnell and J. C. Robson, Noncommutative Noetherian Rings, Graduate Studies in Mathematics, Vol. 30, 2001.

[16] A. I. Ooms and M. Van den Bergh, A degree inequality for Lie algebras with a regular Poisson semi-center, J. Algebra, 323 (2010), 305-322. 
[17] M. Perroud, Projective representations of the Schrödinger group, Helv. Phys. Acta, 50 (2) (1977), 233-252.

[18] L. H. Rowen, Ring Theory, Volume II, Academic Press, INC., 1988.

[19] Y. Wu, L. Zhu, Simple weight modules for Schrödinger algebra, Lin. Alg. Appl., 438 (2013), 559-563.

[20] Y. Wu, Finite dimensional indecomposable modules for Schrödinger algebra, J. Math. Phys., 54, 073503 (2013).

[21] X. Zhang and Y. Cheng, Simple Schrödinger modules which are locally finite over the positive part, J. Pure Appl. Algebra, 219 (2015), 2799-2815.

\section{CONTACT INFORMATION}

Volodymyr Bavula Department of Pure Mathematics, University of Sheffield, Hicks Building, Sheffield S3 7RH, UK

E-Mail(s):v.bavula@sheffield.ac.uk

Tao Lu

School of Mathematical Sciences, Huaqiao University, Quanzhou 362021, Fujian, China

E-Mail(s): lutao.sdu@gmail.com

Received by the editors: 25.01.2021. 